\title{
Plasma technology in fashion and textiles
}

Andrea Zille

2C2T, Centro de Ciência e Tecnologia Têxtil, Departamento de Engenharia Têxtil, Universidade do Minho, Guimarães, Portugal

\subsection{Introduction}

The production of textile materials has grown significantly in recent years. Global fiber production rose to approximately 111 million metric tons in 2018, a rise over the past decade of 35 million tons, and they expect to grow $3.7 \%$ per year to 2025 (Koszewska, 2018). Of the global total, natural fibers accounted for 32 million tons of production during 2018 (cotton production is estimated at 26.72 million tons), an increase of less than 2 million tons in 10 years. The share of natural fibers in global fiber production fell from $41 \%$ in 2008 to less than $30 \%$ in 2018 . Until today the global production of synthetic filament rose to 50 million tons; of this, polyester filament alone was about 45 million tons. Synthetic staple production rose to 22 million tons, and production of cellulosic fibers rose to 7 million tons (Bremen Cotton Report No. 05-06, 2019).

Recently, there have been many efforts at the industrial level to adopt cleaner production processes and technologies. The introduction of a new environmental regulation in European countries called REACH (Registration, Evaluation, Authorisation and Restriction of Chemicals) has forced companies to tackle environmental problems by encouraging innovation with the aim of promoting sustainable development (Europa, 2019). Nowadays, textile industries show clear signs of divergence in the face of decades of stagnation with regard to innovative textile processes and products. The progress of the world textile industry, especially in the processing sector, is linked to the changes coming from new research fields such as micro- and nanotechnology, functionalization, and intelligent fabrics. In this context, plasma technologies emerge as a way to achieve significant improvements in almost all phases of the textile processing, modifying conventional treatments, obtaining important results in rising efficiency, and increasing the durability of functional properties such as capacity for adhesion, dyeing, reactivity, grafting of new chemical groups, and coating of polymers (Zille et al., 2015).

Generally, plasma is produced by applying a flow of voltage over a gas to ionize the atoms/molecules. There exist multiple methods to create ionized gases, in which the following are the typical ones. (a) Glow discharge: Plasma is produced by putting a radio frequency of $40 \mathrm{kHz}, 13.56 \mathrm{MHz}$, microwave, and direct current at low frequency $50 \mathrm{kHz}$ voltage through either a pair or a series of electrodes. This method 
uses reduced pressure and ensure to reach the greatest level of flexibility and uniformity of all plasma usage. (b) Corona discharge: It is known to be producing plasma using atmospheric pressure by putting it through a pair of different size electrodes at a low frequency or high voltage flow. This method is only compatible to certain types of fabrics due to its inability to achieve uniformity (Zille et al. 2014); if not, it could cause some problems. (c) Dielectric-barrier discharge: It is produced using a pulse voltage flow on a pair of electrode, of which one of them is covered in dielectric material. Its biggest advantage that outweighs corona is the ability to reach uniformity.

Plasma technology is based on a simple physical principle. When energy is supplied to matter, it changes state from solids to liquid, and liquids to gaseous. If more energy is supplied to a gas, it becomes ionized and goes into the energy-rich plasma state, the fourth state of matter into which electrons are free from atoms or molecules allowing both species to coexist. Plasma was first discovered by Irving Langmuir in 1928 and is the most common state of matter in the universe. More than $99 \%$ of the visible matter in the universe is in the plasma state (Muthu, 2018).

The development of plasma discharge for application in textile materials is very recent. The first continuous system developed was proposed by Bradley in the year 1971 (Surface Activation Corporation, USA); however, it was a vacuum system, which limited its application due to the high cost involved (Bradley and Fales, 1971). Since then, different systems for application in textile materials have been developed by several manufacturers and research institutes around the world, namely: Sando Iron Works Ltd. (Uzu, Wakayama, Japan); Fraunhofer IGB (Stuttgart, Germany); NIEKMI institute in Russia (Tecnoplasma, S.A); Europlasma (Outdenaarde, Belgium); Polyplas (Emmerthal, Germany); Fourth State (Belmont, USA); Softal Electronic GmbH (Hamburg, Germany); University of Minho (Guimarães, Portugal); Sherman Treater Co (Oxon, UK); Paladin (North Carolina State University); and Dow Corning Plasma Solutions (APGD, AP 100).

There are a large number of plasma types, and an universal classification it is not straightforward; however, they can be primarily divided into thermal and nonthermal plasma (Xi et al., 2008). Thermal plasma can be naturally observed in the stars, lightings, northern lights, other celestial bodies, and the corona of the sun during an eclipse. Thermal plasmas can be also artificially generated using electrical discharges of DC (direct current) or AC (alternate current), laser, radio frequency, and microwave discharges at near-atmospheric pressure (Gleizes et al., 2005). In thermal plasmas, the temperatures are extremely high, in the order of thousands degrees Celsius, and all the different species contained in the gas are in thermal equilibrium.

Since textile materials are heat-sensitive polymers and they cannot withstand the temperature of thermal plasma, nonthermal plasma (or cold plasma) are the only viable option for textile surface modification and processing (Morent et al., 2008). In cold plasmas, the temperature of the electrons is higher $\left(104-105^{\circ} \mathrm{C}\right.$ at $1-10 \mathrm{eV}$ ) than the temperature of other particles (that can remain at room temperature) since the thermodynamic equilibrium is not reached even on a local scale between the electrons and the neutral atoms or molecules, ions and neutral molecules fragments. Cold plasmas, that can be divided into atmospheric pressure plasmas and low-pressure plasmas, have the major advantage of inducing significant surface 
chemical and morphological modifications onto fibrous materials without altering the bulk properties of the materials (Borcia et al., 2005; Oliveira et al., 2010a).

The choice of the process to be applied depends on the processing speed, sample size, and extent of the intended modification (Pappas et al., 2006). Most of the work done to modify polymer surfaces with plasma treatments using different gases and chemicals has been performed at low pressure achieving various effects by etching, polymerization, or formation of free radicals on the surface of the textile substrate (Sarra-Bournet et al., 2006). On one hand, low-pressure plasma technology are considered noncompetitive since the running costs are higher due to the expensive vacuum pumping system and the Meissner trap (cryogenic coil) often required to avoid the water evaporation in the vacuum chamber during unwinding of textiles (Mohammad et al., 2011). These factors have seriously limited the commercial viability of this technique in the textile industry (Pappas et al., 2008). On the other hand, atmospheric cold plasmas are suited because they do not need expensive vacuum equipment and allow continuous and uniform processing of fiber surfaces.

In the last 10 years, plasma technology has become a very active, and high growth research field, assuming a great importance among all available material surface modifications in textile industry. There are several benefits of applying plasma technology, which are best suited with the current vital aspects of sustainability. The benefits are:

- Endless opportunity for the modification of surface properties, by appropriate gases.

- Plasma technology reduces the use of water, chemicals and energy, in comparison with the conventional wet method.

- In terms of economic benefits, the elimination of water sources and chemicals can be more cost-effective.

- Plasma treatment, especially closed plasma process, is environmentally friendly.

The main objective of this chapter is to provide an overview on the most important applications of plasma technology for the fashion and textile industries such as the dyeing and printing processes and the hydrophobic and hydrophilic surface treatments. This chapter also provides a brief description of other areas of application in fashion and textiles, advantages, and drawbacks of the new sustainable technology.

\subsection{Plasma in textile dyeing and printing processes}

Conventional dyeing processes have a low yield, and the dye lost in the effluents can reach up to $50 \%$, creating obvious environmental problems. Most importantly, dye wastewaters without an appropriate treatment can persist in the environment pollution creating problems not only to the photosynthetic processes of the aquatic plants but also to all the living organisms (Schneider et al., 2004). Plasma technology can be used in this context for the removal of the natural or synthetic occurring grease and wax in textile fibers, but also to improve the diffusion of dye molecules into the fibers enhancing color intensities and washing fastness of several natural and synthetic textile materials (Karahan et al., 2008; Nourbakhsh et al., 2008; Souto et al., 2011; Raffaele-Addamo et al., 2006; Hossain et al., 2009; Shahidi et al., 2007; Yaman et al., 2009; Cai and Qiu, 2008; El-Zawahry et al., 2006; Jocic et al., 2005; 
Ratnapandian et al., 2011). Plasma application improves dye exhaustion, dyeing uniformity; decreases the amount of applied dyestuff and water; and allows the reuse of effluents contributing to a significant diminution in costs and environmental impact (Deshmukh and Bhat, 2011; Shah and Shah, 2013; Radetic et al., 2007).

In the last 10 years, atmospheric pressure plasmas have proved to be an effective alternative to low-pressure plasma in dyeing. Excellent results were obtained by dyeing polyamide, polyester, and wool fabrics using different dyestuff such as acid and disperse dyes (Oliveira et al., 2009, 2014; Hossain et al., 2007; Lehocký and Mráček, 2006; Gotoh and Yasukawa, 2010; Gorensek et al., 2009; Mirjalili and Karimi, 2013; Kamel et al., 2011; Salem et al., 2011). The dyeing properties of fibers treated with plasma are correlated with the surface chemical composition and surface modifications (Ren et al., 2011; Xiaoliang et al., 2007; El-Nagar et al., 2006; Gawish et al., 2011; Naebe et al., 2009; Motaghi et al., 2009; Ghoranneviss et al., 2011; Fakin et al., 2009; Ke et al., 2008; Barani and Maleki, 2011). However, the increase in dyeability also depends on the exposure time, gas mixture composition, and applied energy (Kerkeni et al., 2012; Yaman et al., 2011; Carneiro et al., 2005, 2006; Patiño et al., 2011).

In the last 10 years, inkjet printing technologies have demonstrated improved properties over the traditional textile printing methods, such as roller, screen, and transfer printing. These digital technologies are becoming widespread in textile industries displaying excellent quality, low pollution, and very adaptable to the today rapid fashion changes. Despite inkjet printing allows visual effects such as tonal gradients and infinite pattern, the lack of an opportune pretreatment can considerably low the print quality due to the lower capacity to retain water, inks, finish and embossing agents of some textiles. Atmospheric plasma is a very effective pretreatment method to improve inkjet pigment uptake (Fang and Zhang, 2009; Radetic et al., 2000; Kan, 2007; Payamara et al., 2010; Kan et al., 2011; Yuen and Kan, 2007; Zhang and Fang, 2009, 2011; Wang and Wang, 2010; Rashed et al., 2009; Maamoun and Ghalab, 2013; Chvalinova and Wiener, 2008; Nasadil and Benesovsky, 2008).

Overall it is clear that over the last 10 years, atmospheric plasma technologies have significantly improved the dyeing and printing efficiency of textile materials. However, in some cases (e.g., cotton, polyester, and polypropylene fabrics), the low-pressure plasmas remain the most applied technology. Table 6.1 describes the application of plasma in textile dyeing processes by various researchers. Similarly, Table 6.2 and Table 6.3 describe the research studies on hydrophyllic and hydrophobic treatments of textile substrates, respectively.

\subsection{Improving textiles hydrophilicity and hydrophobicity by plasma}

Plasma technology is broadly used to improve surface wettability and/or hydrophilicity of numerous textile materials (Demir et al., 2011; Ren et al., 2008). The increase in hydrophilicity of numerous fibrous materials such as polyamide, polyester, polyethylene, polypropylene, silk, aramid, carbon fibers, wool, and cellulose has been 
Table 6.1 Plasma application in textile dyeing processes.

\begin{tabular}{|c|c|c|c|c|}
\hline $\begin{array}{l}\text { Textile } \\
\text { substrate }\end{array}$ & Carrier gas & $\begin{array}{l}\text { Power } \\
\text { (W) }\end{array}$ & Dye (C.I.) & References \\
\hline \multicolumn{5}{|c|}{ Glow discharge (APGD) } \\
\hline Polyester & Air & n.a. & Disperse Red 127 & $\begin{array}{l}\text { Gotoh and } \\
\text { Yasukawa } \\
\text { (2010) }\end{array}$ \\
\hline Polyester & Air & n.a. & $\begin{array}{l}\text { Disperse Yellow } \\
211\end{array}$ & $\begin{array}{l}\text { Gotoh and } \\
\text { Yasukawa } \\
(2010)\end{array}$ \\
\hline Polyester & Air & n.a. & Disperse Violet 57 & $\begin{array}{l}\text { Gotoh and } \\
\text { Yasukawa } \\
\text { (2010) }\end{array}$ \\
\hline Wool & $\mathrm{He}$ & 5000 & Acid Red 13 & Naebe et al. (2009) \\
\hline Wool & $\mathrm{He}$ & 5000 & Reactive Red 84 & Naebe et al. (2009) \\
\hline Wool & $\mathrm{He}$ & 5000 & Natural Caspian & $\begin{array}{l}\text { Ratnapandian et al. } \\
\text { (2011) }\end{array}$ \\
\hline Wool & $\mathrm{He}+\mathrm{N}_{2}$ & 5000 & Natural Caspian & $\begin{array}{l}\text { Ratnapandian et al. } \\
\text { (2011) }\end{array}$ \\
\hline Wool & $\mathrm{He}$ & 5000 & Natural Thar & $\begin{array}{l}\text { Ratnapandian et al. } \\
\text { (2011) }\end{array}$ \\
\hline Wool & $\mathrm{He}+\mathrm{N}_{2}$ & 5000 & Natural Thar & $\begin{array}{l}\text { Ratnapandian et al. } \\
\text { (2011) }\end{array}$ \\
\hline Wool & $\mathrm{Air}+\mathrm{He}$ & n.a. & Acid Blue 113 & $\begin{array}{l}\text { Cai and Qiu } \\
\text { (2008) }\end{array}$ \\
\hline Wool & $\mathrm{O}_{2}+\mathrm{He}$ & n.a. & Acid Blue 113 & $\begin{array}{l}\text { Cai and Qiu } \\
\text { (2008) }\end{array}$ \\
\hline \multicolumn{5}{|c|}{ Plasma corona } \\
\hline Cotton & Air & 380 & Reactive Blue & Patiño et al. (2011) \\
\hline Cotton & Air & 660 & Reactive Black 5 & $\begin{array}{l}\text { Nourbakhsh et al. } \\
\text { (2008) }\end{array}$ \\
\hline Cotton & Air & 660 & Reactive Red 158 & $\begin{array}{l}\text { Nourbakhsh et al. } \\
\text { (2008) }\end{array}$ \\
\hline Cotton & Air & 1000 & Direct Red 80 & $\begin{array}{l}\text { Carneiro et al. } \\
\text { (2001) }\end{array}$ \\
\hline Cotton & Air & 1000 & Direct Red 243 & $\begin{array}{l}\text { Carneiro et al. } \\
\text { (2001) }\end{array}$ \\
\hline
\end{tabular}


Table 6.1 Continued

\begin{tabular}{|c|c|c|c|c|}
\hline $\begin{array}{l}\text { Textile } \\
\text { substrate }\end{array}$ & Carrier gas & $\begin{array}{l}\text { Power } \\
\text { (W) }\end{array}$ & Dye (C.I.) & References \\
\hline Cotton & Air & 900 & Disperse Red 73 & $\begin{array}{l}\text { Gorensek et al. } \\
\text { (2009) }\end{array}$ \\
\hline Cotton & Air & 6000 & Disperse Blue 79 & $\mathrm{Xu}$ and Liu (2003) \\
\hline Cotton & $\mathrm{O}_{2}+\mathrm{N}_{2}$ & 2500 & Acid Blue 158 & Fakin et al. (2009) \\
\hline Cotton & $\mathrm{O}_{2}+\mathrm{N}_{2}$ & 2500 & Acid Blue 113 & Fakin et al. (2009) \\
\hline \multicolumn{5}{|c|}{ Dielectric barrier discharge plasma (DBD) } \\
\hline Cotton & Air + TETA & 100 & Acid Red 99 & $\begin{array}{l}\text { Karahan et al. } \\
\text { (2008) }\end{array}$ \\
\hline Cotton & $\mathrm{Ar}+\mathrm{TETA}$ & 100 & Acid Red 99 & $\begin{array}{l}\text { Karahan et al. } \\
\quad(2008)\end{array}$ \\
\hline Polyamide & Air & 1000 & Direct Orange 57 & $\begin{array}{l}\text { Oliveira et al. } \\
\text { (2014) }\end{array}$ \\
\hline Polyamide & Air & 1000 & Direct Orange 57 & Souto et al. (2012) \\
\hline Polyamide & Air & 1500 & Reactive Yellow 27 & Souto et al. (2011) \\
\hline Polyamide & Air & 1500 & Reactive Red 231 & Souto et al. (2011) \\
\hline Polyamide & Air & 1500 & Reactive Yellow 26 & Souto et al. (2011) \\
\hline Polyamide & Air & 1500 & Realan Red EHF® & Souto et al. (2011) \\
\hline Polyamide & Air & 1500 & $\begin{array}{l}\text { Realan Yellow } \\
\text { EHF® }\end{array}$ & Souto et al. (2011) \\
\hline Polyamide & Air & 1500 & Realan Blue EHF® & Souto et al. (2011) \\
\hline Polyamide & Air & 1500 & Sirius Scarlet $\mathrm{KCF}{ }^{\circledR}$ & Souto et al. (2011) \\
\hline Polyamide & Air & 1500 & Direct Violet 47 & Souto et al. (2011) \\
\hline Polyamide & Air & 1500 & Direct Orange 57 & Souto et al. (2011) \\
\hline Polyamide & Air & 1500 & $\begin{array}{l}\text { Telon Blue } \\
\text { MGWL® }\end{array}$ & Souto et al. (2011) \\
\hline Polyamide & Air & 1500 & Telon Red A2FR & Souto et al. (2011) \\
\hline Polyamide & Air & 1500 & Telon Rot M-6BW & Souto et al. (2011) \\
\hline Polyamide & Air & 600 & $\begin{array}{l}\text { Reactive Yellow } \\
125\end{array}$ & $\begin{array}{l}\text { Oliveira et al. } \\
\text { (2010b) }\end{array}$ \\
\hline Polyamide & Air & 600 & Acid yellow 240 & $\begin{array}{l}\text { Oliveira et al. } \\
\text { (2010b) }\end{array}$ \\
\hline Polyamide & Air & 30 & Disperse Blue 19 & $\begin{array}{l}\text { Kamel et al. } \\
\text { (2011) }\end{array}$ \\
\hline
\end{tabular}


Table 6.1 Continued

\begin{tabular}{|c|c|c|c|c|}
\hline $\begin{array}{l}\text { Textile } \\
\text { substrate }\end{array}$ & Carrier gas & $\begin{array}{l}\text { Power } \\
\text { (W) }\end{array}$ & Dye (C.I.) & References \\
\hline Polyamide & Air & 30 & Disperse Brown 1 & $\begin{array}{l}\text { Kamel et al. } \\
\qquad(2011)\end{array}$ \\
\hline Polyamide & $\mathrm{N}_{2}$ & 30 & Disperse Blue 19 & $\begin{array}{l}\text { Kamel et al. } \\
\qquad(2011)\end{array}$ \\
\hline Polyamide & $\mathrm{N}_{2}$ & 30 & Disperse Brown 1 & $\begin{array}{l}\text { Kamel et al. } \\
\text { (2011) }\end{array}$ \\
\hline Polyamide & $\mathrm{O}_{2}$ & 30 & Disperse Blue 19 & $\begin{array}{l}\text { Kamel et al. } \\
\text { (2011) }\end{array}$ \\
\hline Polyamide & $\mathrm{O}_{2}$ & 30 & Disperse Brown 1 & $\begin{array}{l}\text { Kamel et al. } \\
\qquad(2011)\end{array}$ \\
\hline Polyamide & $\mathrm{Ar}$ & 30 & Disperse Blue 19 & $\begin{array}{l}\text { Kamel et al. } \\
\text { (2011) }\end{array}$ \\
\hline Polyamide & Ar & 30 & Disperse Brown 1 & $\begin{array}{l}\text { Kamel et al. } \\
\text { (2011) }\end{array}$ \\
\hline Polyamide & Air & 1000 & Curcumin & $\begin{array}{c}\text { Kerkeni et al. } \\
\text { (2012) }\end{array}$ \\
\hline Polypropylene & $\mathrm{Ar}$ & 100 & Vat Yellow 46 & $\begin{array}{l}\text { Yaman et al. } \\
\text { (2011) }\end{array}$ \\
\hline Polypropylene & Air & 100 & Vat Yellow 46 & $\begin{array}{c}\text { Yaman et al. } \\
\text { (2011) }\end{array}$ \\
\hline Polypropylene & Air & 130 & Basic Yellow 28 & $\begin{array}{c}\text { Yaman et al. } \\
\text { (2013) }\end{array}$ \\
\hline Polypropylene & Air & 130 & Basic Red 18 & $\begin{array}{c}\text { Yaman et al. } \\
\text { (2013) }\end{array}$ \\
\hline Polypropylene & Air & 130 & Basic Red 46 & $\begin{array}{c}\text { Yaman et al. } \\
\text { (2013) }\end{array}$ \\
\hline Polypropylene & $\mathrm{Ar}$ & 200 & Basic Blue 3 & $\begin{array}{c}\text { Yaman et al. } \\
\text { (2009) }\end{array}$ \\
\hline Polypropylene & $\mathrm{Ar}$ & 200 & Basic Red 18 & $\begin{array}{c}\text { Yaman et al. } \\
\text { (2009) }\end{array}$ \\
\hline Polypropylene & $\mathrm{Ar}$ & 200 & Basic Red 46 & $\begin{array}{c}\text { Yaman et al. } \\
\text { (2009) }\end{array}$ \\
\hline Polypropylene & Ar & 200 & Acid Blue 264 & $\begin{array}{c}\text { Yaman et al. } \\
\text { (2009) }\end{array}$ \\
\hline Polypropylene & $\mathrm{Ar}$ & 200 & Acid Violet 17 & $\begin{array}{c}\text { Yaman et al. } \\
\text { (2009) }\end{array}$ \\
\hline
\end{tabular}


Table 6.1 Continued

\begin{tabular}{|c|c|c|c|c|}
\hline $\begin{array}{l}\text { Textile } \\
\text { substrate }\end{array}$ & Carrier gas & $\begin{array}{l}\text { Power } \\
(\mathbf{W})\end{array}$ & Dye (C.I.) & References \\
\hline Polypropylene & Air & 600 & $\begin{array}{l}\text { Reactive Yellow } \\
125\end{array}$ & $\begin{array}{l}\text { Oliveira et al. } \\
\text { (2010b) }\end{array}$ \\
\hline Polypropylene & Air & 600 & Acid yellow 240 & $\begin{array}{l}\text { Oliveira et al. } \\
\text { (2010b) }\end{array}$ \\
\hline Polypropylene & $\mathrm{N}_{2}$ & 30 & Acid Orange 19 & $\begin{array}{l}\text { El-Zawahry et al. } \\
\text { (2006) }\end{array}$ \\
\hline Polypropylene & $\mathrm{N}_{2}$ & 30 & Acid Green 9 & $\begin{array}{l}\text { El-Zawahry et al. } \\
\text { (2006) }\end{array}$ \\
\hline Polypropylene & $\mathrm{N}_{2}$ & 30 & Acid Red 249 & $\begin{array}{l}\text { El-Zawahry et al. } \\
\text { (2006) }\end{array}$ \\
\hline Polypropylene & $\mathrm{N}_{2}$ & 30 & Acid Blue 83 & $\begin{array}{l}\text { El-Zawahry et al. } \\
\text { (2006) }\end{array}$ \\
\hline Polypropylene & $\mathrm{N}_{2}$ & 30 & Acid Blue 25 & $\begin{array}{l}\text { El-Zawahry et al. } \\
\text { (2006) }\end{array}$ \\
\hline Polypropylene & $\mathrm{N}_{2}$ & 30 & Acid Green 27 & $\begin{array}{l}\text { El-Zawahry et al. } \\
\text { (2006) }\end{array}$ \\
\hline Polypropylene & $\mathrm{N}_{2}$ & 30 & Reactive Blue 19 & $\begin{array}{l}\text { El-Zawahry et al. } \\
\text { (2006) }\end{array}$ \\
\hline Polypropylene & $\mathrm{N}_{2}$ & 30 & Reactive Red 4 & $\begin{array}{l}\text { El-Zawahry et al. } \\
\text { (2006) }\end{array}$ \\
\hline Polypropylene & $\mathrm{N}_{2}$ & 30 & Reactive Red 194 & $\begin{array}{l}\text { El-Zawahry et al. } \\
\text { (2006) }\end{array}$ \\
\hline Polypropylene & $\mathrm{N}_{2}$ & 30 & Reactive Black 5 & $\begin{array}{l}\text { El-Zawahry et al. } \\
\text { (2006) }\end{array}$ \\
\hline Polypropylene & $\mathrm{N}_{2}$ & 30 & Reactive red 120 & $\begin{array}{l}\text { El-Zawahry et al. } \\
\text { (2006) }\end{array}$ \\
\hline \multicolumn{5}{|c|}{ Low-pressure plasma (LPP) } \\
\hline Polypropylene & EDA & 20 & Reactive Black 5 & $\begin{array}{l}\text { Ozdogan et al. } \\
\text { (2002) }\end{array}$ \\
\hline Polypropylene & TETA & 20 & Reactive Black 5 & $\begin{array}{l}\text { Ozdogan et al. } \\
\text { (2002) }\end{array}$ \\
\hline Polypropylene & $\mathrm{Ar}+\mathrm{EDA}$ bath & 20 & Reactive Black 5 & $\begin{array}{l}\text { Ozdogan et al. } \\
\text { (2002) }\end{array}$ \\
\hline Polypropylene & Ar + TETA bath & 20 & Reactive Black 5 & $\begin{array}{l}\text { Ozdogan et al. } \\
\text { (2002) }\end{array}$ \\
\hline
\end{tabular}


Table 6.1 Continued

\begin{tabular}{|c|c|c|c|c|}
\hline $\begin{array}{l}\text { Textile } \\
\text { substrate }\end{array}$ & Carrier gas & $\begin{array}{l}\text { Power } \\
\text { (W) }\end{array}$ & Dye (C.I.) & References \\
\hline Polypropylene & Air & 40 & Reactive Red 1 & Bhat et al. (2011) \\
\hline Polypropylene & Air & 40 & Direct Red 28 & Bhat et al. (2011) \\
\hline Polypropylene & Air & 40 & Natural Amazon & Bhat et al. (2011) \\
\hline Polypropylene & $\mathrm{O}_{2}$ & 120 & Basic Blue 9 & $\begin{array}{l}\text { Malek and Holme } \\
\quad(2003)\end{array}$ \\
\hline Polypropylene & Air & 20 & Direct Red 81 & $\begin{array}{l}\text { Malek and Holme } \\
\text { (2003) }\end{array}$ \\
\hline Polypropylene & $\mathrm{O}_{2}$ & 70 & Direct Red 81 & $\begin{array}{l}\text { Malek and Holme } \\
\text { (2003) }\end{array}$ \\
\hline Polypropylene & $\mathrm{O}_{2}$ & 120 & Direct Red 81 & $\begin{array}{l}\text { Malek and Holme } \\
\quad(2003)\end{array}$ \\
\hline Polypropylene & $\mathrm{O}_{2}$ & 300 & Acid Red 330 & $\begin{array}{l}\text { Sun and Stylios } \\
\text { (2004) }\end{array}$ \\
\hline Polypropylene & Acrylic acid & 10 & Basic red 18 & $\begin{array}{l}\text { Oktem et al. } \\
\text { (2000) }\end{array}$ \\
\hline Polypropylene & Water & 10 & Basic red 18 & $\begin{array}{l}\text { Oktem et al. } \\
\text { (2000) }\end{array}$ \\
\hline Polypropylene & $\mathrm{Ar}$ & 10 & Basic red 18 & $\begin{array}{l}\text { Oktem et al. } \\
\text { (2000) }\end{array}$ \\
\hline Polypropylene & Air & 10 & Basic red 18 & $\begin{array}{l}\text { Oktem et al. } \\
\text { (2000) }\end{array}$ \\
\hline Polypropylene & $\mathrm{O}_{2}$ & 10 & Basic red 18 & $\begin{array}{l}\text { Oktem et al. } \\
\text { (2000) }\end{array}$ \\
\hline Polypropylene & Acrylic acid & 10 & Basic red 24 & $\begin{array}{l}\text { Ferrero et al. } \\
\text { (2004) }\end{array}$ \\
\hline Polypropylene & $\begin{array}{l}\mathrm{O}_{2}+\text { acrylic acid } \\
\text { bath }\end{array}$ & 800 & Acid dye & Liao et al. (2005) \\
\hline Polypropylene & $\mathrm{O}_{2}+$ HEMA bath & 800 & Acid dye & Liao et al. (2005) \\
\hline Polypropylene & $\mathrm{CF}_{4}$ & 100 & Acid Blue 62 & Yip et al. (2002) \\
\hline Polypropylene & $\mathrm{CF}_{4}$ & 100 & Disperse Blue 56 & Yip et al. (2002) \\
\hline Polypropylene & Acrylic acid & 20 & Basic red 18 & Oktem et al. (1999) \\
\hline Polypropylene & $\begin{array}{l}\text { Ar }+ \text { acrylic acid } \\
\quad \text { bath }\end{array}$ & 20 & Basic red 18 & Oktem et al. (1999) \\
\hline
\end{tabular}


Table 6.1 Continued

\begin{tabular}{|c|c|c|c|c|}
\hline $\begin{array}{l}\text { Textile } \\
\text { substrate }\end{array}$ & Carrier gas & $\begin{array}{l}\text { Power } \\
(\mathrm{W})\end{array}$ & Dye (C.I.) & References \\
\hline Polypropylene & $\mathrm{N}_{2}$ & n.a. & Disperse Red 60 & $\begin{array}{l}\text { Mirjalili and } \\
\text { Karimi (2013) }\end{array}$ \\
\hline Polypropylene & $\mathrm{N}_{2}$ & n.a. & Acid Red 138 & $\begin{array}{l}\text { Mirjalili and } \\
\text { Karimi (2013) }\end{array}$ \\
\hline Polypropylene & $\mathrm{N}_{2}$ & n.a. & Basic Violet 16 & $\begin{array}{l}\text { Mirjalili and } \\
\text { Karimi (2013) }\end{array}$ \\
\hline Polypropylene & $\begin{array}{l}\mathrm{O}_{2}+\text { poly- } \\
\text { DADMAC }\end{array}$ & 600 & Acid Red 18 & Salem et al. (2011) \\
\hline Polypropylene & $\begin{array}{l}\mathrm{O}_{2}+\text { poly- } \\
\text { DADMAC }\end{array}$ & 600 & Acid Blue 80 & Salem et al. (2011) \\
\hline Polypropylene & $\mathrm{NH}_{3}+\mathrm{C}_{2} \mathrm{H}_{4}$ & 600 & Acid Blue 127 & $\begin{array}{l}\text { Hossain et al. } \\
\text { (2007) }\end{array}$ \\
\hline Polypropylene & Air & 10 & Direct Red 79 & $\begin{array}{l}\text { Lehocký and } \\
\text { Mráček (2006) }\end{array}$ \\
\hline Polypropylene & $\mathrm{NH}_{3}+\mathrm{C}_{2} \mathrm{H}_{2}$ & 475 & Acid Blue 127 & $\begin{array}{l}\text { Hossain et al. } \\
\text { (2009) }\end{array}$ \\
\hline Polypropylene & Acrylic acid & 10 & Basic red 18 & $\begin{array}{l}\text { Oktem et al. } \\
\text { (2000) }\end{array}$ \\
\hline Polypropylene & Water & 10 & Basic red 18 & $\begin{array}{l}\text { Oktem et al. } \\
\text { (2000) }\end{array}$ \\
\hline Polypropylene & $\mathrm{Ar}$ & 10 & Basic red 18 & $\begin{array}{l}\text { Oktem et al. } \\
\text { (2000) }\end{array}$ \\
\hline Polypropylene & Air & 10 & Basic red 18 & $\begin{array}{l}\text { Oktem et al. } \\
\qquad(2000)\end{array}$ \\
\hline Polypropylene & $\mathrm{O}_{2}$ & 10 & Basic red 18 & $\begin{array}{l}\text { Oktem et al. } \\
\text { (2000) }\end{array}$ \\
\hline Polypropylene & Acrylic acid & 25 & Basic red 24 & $\begin{array}{l}\text { Ferrero et al. } \\
\quad(2004)\end{array}$ \\
\hline Polypropylene & Air & 120 & Disperse Blue 94 & $\begin{array}{l}\text { Raffaele-Addamo } \\
\text { et al. (2006) }\end{array}$ \\
\hline Polypropylene & $\mathrm{Ar}$ & 130 & Disperse Blue 94 & $\begin{array}{l}\text { Raffaele-Addamo } \\
\text { et al. (2006) }\end{array}$ \\
\hline Polypropylene & $\mathrm{SiCl}_{4}$ & n.a. & Basic Blue & $\begin{array}{l}\text { Sarmadi et al. } \\
\text { (1996) }\end{array}$ \\
\hline
\end{tabular}


Table 6.1 Continued

\begin{tabular}{|c|c|c|c|c|}
\hline $\begin{array}{l}\text { Textile } \\
\text { substrate }\end{array}$ & Carrier gas & $\begin{array}{l}\text { Power } \\
(\mathbf{W})\end{array}$ & Dye (C.I.) & References \\
\hline PET/Cotton & Acrylic acid & 10 & Basic red 18 & $\begin{array}{l}\text { Oktem et al. } \\
\text { (2002) }\end{array}$ \\
\hline PET/Cotton & Water & 10 & Basic red 18 & $\begin{array}{l}\text { Oktem et al. } \\
\text { (2002) }\end{array}$ \\
\hline PET/Cotton & Acrylic acid & 75 & Basic red 24 & $\begin{array}{l}\text { Ferrero et al. } \\
\qquad(2004)\end{array}$ \\
\hline PET/Cotton & $\mathrm{Ar}+$ acrylonitrile & 100 & Basic dye & $\begin{array}{l}\text { Sarmadi et al. } \\
\text { (1993) }\end{array}$ \\
\hline Silk & $\mathrm{O}_{2}$ & 50 & Reactive Black 5 & $\begin{array}{l}\text { Iriyama et al. } \\
\text { (2002) }\end{array}$ \\
\hline Silk & $\mathrm{N}_{2}$ & 50 & Reactive Black 5 & $\begin{array}{l}\text { Iriyama et al. } \\
\text { (2002) }\end{array}$ \\
\hline Silk & $\mathrm{H}_{2}$ & 50 & Reactive Black 5 & $\begin{array}{l}\text { Iriyama et al. } \\
\text { (2002) }\end{array}$ \\
\hline Silk & $\mathrm{O}_{2}$ & 20 & Acid Orange 19 & $\begin{array}{l}\text { Gawish et al. } \\
\text { (2011) }\end{array}$ \\
\hline Silk & $\mathrm{O}_{2}$ & 20 & Acid Violet 90 & $\begin{array}{l}\text { Gawish et al. } \\
\text { (2011) }\end{array}$ \\
\hline Silk & $\mathrm{O}_{2}$ & 20 & Reactive Red 194 & $\begin{array}{l}\text { Gawish et al. } \\
\text { (2011) }\end{array}$ \\
\hline Silk & Air & 100 & Acid Red 27 & $\begin{array}{l}\text { Jocic et al. } \\
\text { (2005) }\end{array}$ \\
\hline Silk & $\mathrm{N}_{2}$ & 40 & Acid Blue 83 & $\begin{array}{l}\text { El-Zawahry et al. } \\
\text { (2006) }\end{array}$ \\
\hline Silk & $\mathrm{N}_{2}$ & 40 & Acid Red 249 & $\begin{array}{l}\text { El-Zawahry et al. } \\
\text { (2006) }\end{array}$ \\
\hline Silk & $\mathrm{N}_{2}$ & 50 & Reactive Blue 50 & $\begin{array}{l}\text { Jin and Dai } \\
\text { (2003) }\end{array}$ \\
\hline Silk & $\mathrm{O}_{2}$ & 300 & Acid Red 330 & $\begin{array}{l}\text { Sun and Stylios } \\
\text { (2004) }\end{array}$ \\
\hline
\end{tabular}

demonstrated (Riccardi et al., 2005; Masaeli et al., 2007; Li et al., 2009; Huang et al., 2013; Bessada et al., 2011; Simor et al., 2010; Wang and Qiu, 2007; Vander Wielen et al., 2006). Plasma treatments in air or using different carrier gases are able to introduce hydrophilic functional groups such as $-\mathrm{COOH},-\mathrm{OH}$, and $-\mathrm{NH}_{2}$ on the fabric surface. The increase in wettability is attributed to the generation of these new 
Table 6.2 Plasma technology in hydrophyllic treatment of textiles.

\begin{tabular}{|c|c|c|c|}
\hline Textile substrate & Carrier gas & Power $(\mathbf{W})$ & References \\
\hline \multicolumn{4}{|c|}{ Glow discharge (APGD) } \\
\hline Cotton & Air & $200^{\mathrm{a}}$ & Temmerman and Leys (2005) \\
\hline PA6 & $\mathrm{N}_{2}, \mathrm{C}_{2} \mathrm{H}_{2}-\mathrm{He}$ & 850 & Pappas et al. (2006) \\
\hline PA6 & $\mathrm{He}$ & 50 & Samanta et al. (2009) \\
\hline PET & $\mathrm{He}$ & 50 & Samanta et al. (2009) \\
\hline PET & $\mathrm{Ar}$ & $0.3^{\mathrm{a}}$ & Kabajev and Prosycevas (2004) \\
\hline PET & Air & $0.3^{\mathrm{a}}$ & Kabajev and Prosycevas (2004) \\
\hline PET & $\mathrm{O}_{2}$ & $0.3^{\mathrm{a}}$ & Kabajev and Prosycevas (2004) \\
\hline Polylinen ${ }^{\circledR}$ & $\mathrm{O}_{2}$ & 460 & Karthik et al. (2013) \\
\hline $\mathrm{PP}$ & $\mathrm{He}$ & $0.005^{\mathrm{c}}$ & Hwang et al. (2005) \\
\hline Wool & Air & 150 & Binias et al. (2004) \\
\hline Wool & $\mathrm{C}_{3} \mathrm{H}_{4} \mathrm{O}_{2}$ & $40-100$ & Kutlu et al. (2010) \\
\hline \multicolumn{4}{|c|}{ Atmospheric pressure plasma jet (APPJ) } \\
\hline Cotton & $\mathrm{O}_{2}-\mathrm{He}$ & 40 & Tian et al. (2011) \\
\hline Cotton & $\mathrm{O}_{2}-\mathrm{He}$ & 40 & Sun and Qiu (2012) \\
\hline Cotton (hexane) & $\mathrm{O}_{2}-\mathrm{He}$ & 40 & Tian et al. (2011) \\
\hline PA & $\mathrm{O}_{2}-\mathrm{He}$ & $13.6^{\mathrm{c}}$ & Kan and Yuen (2013) \\
\hline PET & $\mathrm{Ar}$ & $14-20$ & Cheng et al. (2006) \\
\hline PET & $\mathrm{O}_{2}-\mathrm{He}$ & $13.6^{\mathrm{c}}$ & Kan and Yuen (2013) \\
\hline PET & $\mathrm{O}_{2}-\mathrm{He}$ & 100 & Wang et al. (2008) \\
\hline PET filament & Air & n.a. & Gotoh and Yasukawa (2010) \\
\hline PET spun & Air & n.a. & Gotoh and Yasukawa (2010) \\
\hline $\mathrm{PP}$ & $\mathrm{Ar}$ & $14-20^{\mathrm{b}}$ & Cheng et al. (2006) \\
\hline Wool & $\mathrm{O}_{2}-\mathrm{He}$ & 40 & Wang and Qiu (2007) \\
\hline Wool & $\mathrm{O}_{2}-\mathrm{He}$ & 40 & Wang and Qiu (2012) \\
\hline Wool & $\mathrm{O}_{2}-\mathrm{He}$ & $13.56^{\mathrm{c}}$ & Cheng et al. (2010) \\
\hline \multicolumn{4}{|l|}{ Plasma corona } \\
\hline Cotton & Air & 530 & Carneiro et al. (2001) \\
\hline PA6 & Air & n.a. & Ryu et al. (1991) \\
\hline PET & Air & n.a. & Ryu et al. (1991) \\
\hline PET & Air & $7.1^{\mathrm{a}}$ & Pichal et al. (2004) \\
\hline PET & Air & 6000 & Xu and Liu (2003) \\
\hline Wool & Air & n.a. & Ryu et al. (1991) \\
\hline
\end{tabular}


Table 6.2 Continued

\begin{tabular}{|c|c|c|c|}
\hline Textile substrate & Carrier gas & Power $(\mathbf{W})$ & References \\
\hline \multicolumn{4}{|c|}{ Dielectric barrier discharge plasma (DBD) } \\
\hline Acryclic & Air & 600 & Oliveira et al. (2010b) \\
\hline Aramid & Air & $23.33^{\mathrm{a}}$ & Xi et al. (2008) \\
\hline Aramid & Air & $33.33^{\mathrm{a}}$ & Xi et al. (2008) \\
\hline Aramid & Air & $27.6^{\mathrm{a}}$ & Jia et al. (2011) \\
\hline Carbon fibers & Air & $0.008^{c}$ & Li et al. (2009) \\
\hline Cotton & Air & 50 & Karahan and Özdogan (2008) \\
\hline Cotton & Air & 100 & Karahan and Özdogan (2008) \\
\hline Cotton & Air & 130 & Karahan and Özdogan (2008) \\
\hline Cotton & $\mathrm{Ar}$ & 50 & Karahan and Özdogan (2008) \\
\hline Cotton & $\mathrm{Ar}$ & 100 & Karahan and Özdogan (2008) \\
\hline Cotton & $\mathrm{Ar}$ & 130 & Karahan and Özdogan (2008) \\
\hline Diacetate & Air & 600 & Oliveira et al. (2010b) \\
\hline NCE & Air & $5^{\mathrm{b}}$ & Borcia et al. (2006) \\
\hline PA & Air & 600 & Oliveira et al. (2010b) \\
\hline PA & Air & $5^{\mathrm{b}}$ & Borcia et al. (2006) \\
\hline PA12 & Air & $5.7^{\mathrm{b}}$ & Upadhyay et al. (2004) \\
\hline PA6 & Air & $5.7^{\mathrm{b}}$ & Upadhyay et al. (2004) \\
\hline PA6 & $\mathrm{He}-\mathrm{O}_{2}$ & 1200 & Pappas et al. (2008) \\
\hline PA66 & Air & $2400^{\mathrm{a}}$ & Souto et al. (2011) \\
\hline PA66 & Air & $500-1500$ & Bessada et al. (2011) \\
\hline $\mathrm{PE}$ & Air & $2^{\mathrm{a}}$ & Akishev et al. (2003) \\
\hline $\mathrm{PE}$ & $\mathrm{N}_{2}$ & $0.7^{\mathrm{a}}$ & Akishev et al. (2003) \\
\hline PE & Ar & $0.12^{\mathrm{a}}$ & Akishev et al. (2003) \\
\hline PE & $\mathrm{He}-\mathrm{O}_{2}$ & 1200 & Pappas et al. (2008) \\
\hline PE & Air & 6 & De Geyter et al. (2008) \\
\hline PET & Air & $2^{\mathrm{a}}$ & Akishev et al. (2003) \\
\hline PET & $\mathrm{Ar}$ & $0.12^{\mathrm{a}}$ & Akishev et al. (2003) \\
\hline PET & Air & 600 & Oliveira et al. (2010b) \\
\hline PET & Air & $5^{\mathrm{b}}$ & Borcia et al. (2006) \\
\hline PET & Air & $50^{\mathrm{b}}$ & Morent et al. (2007) \\
\hline
\end{tabular}


Table 6.2 Continued

\begin{tabular}{|c|c|c|c|}
\hline Textile substrate & Carrier gas & Power $(W)$ & References \\
\hline PET & $\mathrm{He}$ & $50^{\mathrm{b}}$ & Morent et al. (2007) \\
\hline PET & $\mathrm{Ar}$ & $50^{\mathrm{b}}$ & Morent et al. (2007) \\
\hline PET & Air & n.a. & Klenko et al. (2006) \\
\hline PET & Air & $500-1500$ & Bessada et al. (2011) \\
\hline PET & Air & $500^{\mathrm{b}}$ & Leroux et al. (2009) \\
\hline PI & $\mathrm{He}-\mathrm{O}_{2}$ & 1200 & Pappas et al. (2008) \\
\hline PP & Air & $2^{\mathrm{a}}$ & Akishev et al. (2003) \\
\hline PP & $\mathrm{N}_{2}$ & $0.7^{\mathrm{a}}$ & Akishev et al. (2003) \\
\hline PP & $\mathrm{Ar}$ & $0.12^{\mathrm{a}}$ & Akishev et al. (2003) \\
\hline PP & Air & $200^{\mathrm{b}}$ & Morent et al. (2007) \\
\hline PP & $\mathrm{He}$ & $200^{\mathrm{b}}$ & Morent et al. (2007) \\
\hline $\mathrm{PP}$ & Ar & $200^{\mathrm{b}}$ & Morent et al. (2007) \\
\hline PP & $\mathrm{N}_{2}$ & $0.5^{\mathrm{b}}$ & Rahel' et al. (2003) \\
\hline PTFE & $\mathrm{He}-\mathrm{O}_{2}$ & 1200 & Pappas et al. (2008) \\
\hline Wool & Air & 600 & Oliveira et al. (2010b) \\
\hline Wool & Air & $5^{\mathrm{b}}$ & Borcia et al. (2006) \\
\hline \multicolumn{4}{|c|}{ Low-pressure plasma (LPP) } \\
\hline Aramid & $\mathrm{Ar}$ & n.a. & Freitas et al. (2006) \\
\hline Aramid & $\mathrm{N}_{2}$ & n.a. & Freitas et al. (2006) \\
\hline Aramid & $\mathrm{O}_{2}$ & n.a. & Freitas et al. (2006) \\
\hline Aramid & $\mathrm{O}_{2}$ & $0.38^{\mathrm{a}}$ & Verschuren (2005) \\
\hline Cotton & $\mathrm{O}_{2}$ & $0.64^{\mathrm{a}}$ & Poll et al. (2001) \\
\hline Cotton & $\mathrm{O}_{2}$ & 300 & Sun and Stylios (2004) \\
\hline Lyocell® & $\mathrm{O}_{2}$ & 500 & Persin et al. (2012) \\
\hline Modal ${ }^{\circledR}$ & $\mathrm{O}_{2}$ & 500 & Persin et al. (2012) \\
\hline PA6 & $\mathrm{N}_{2}$ & 60 & Canal et al. (2007) \\
\hline PA6 & $\mathrm{O}_{2}$ & 60 & Canal et al. (2007) \\
\hline PE & $\mathrm{Ar}$ & 40 & Huang et al. (2013) \\
\hline PET & $\mathrm{SiCl}_{4}$ & 150 & Negulescu et al. (2000) \\
\hline PET & $\mathrm{O}_{2}, \mathrm{~N}_{2}, \mathrm{H}$ & n.a. & Costa et al. (2006) \\
\hline PET & $\mathrm{O}_{2}, \mathrm{~N}_{2}$ & $0.4^{\mathrm{b}}$ & Vatuňa et al. (2004) \\
\hline PET & Air & $7.7^{\mathrm{b}}$ & Riccardi et al. (2003) \\
\hline
\end{tabular}


Table 6.2 Continued

\begin{tabular}{|l|l|l|l|}
\hline Textile substrate & Carrier gas & Power $(\mathbf{W})$ & References \\
\hline PET & $\mathrm{O}_{2}$ & $0.38^{\mathrm{a}}$ & Verschuren (2005) \\
PP & $\mathrm{O}_{2}$ & 300 & Wei et al. (2005) \\
PP & $\mathrm{O}_{2}$ & 500 & Masaeli et al. (2007) \\
Viscose & $\mathrm{O}_{2}$ & 500 & Persin et al. (2012) \\
Wool & $\mathrm{O}_{2}$ & 300 & Sun and Stylios (2004) \\
Wool & $\mathrm{N}_{2}$ & 60 & Canal et al. (2007) \\
Wool & $\mathrm{O}_{2}-\mathrm{N}_{2}$ & 60 & Canal et al. (2007) \\
Wool & $\mathrm{O}_{2}$ & 60 & Canal et al. (2007) \\
\hline
\end{tabular}

n.a., not available.

${ }^{\mathrm{a}}$ Power density $\left(\mathrm{W} \mathrm{cm}^{-2}\right)$.

${ }^{\mathrm{b}}$ Power (W).

${ }^{\mathrm{c}}$ Frequency $(\mathrm{MHz})$.

chemical groups and/or to the reduction/elimination of polymeric layers on the fabric surface. The wettability of a textile material is directly related to its surface energy and defines surface and interfacial phenomena including chemical reactivity, adsorption, desorption, wet processing, and adhesion (Kale and Desai, 2011).

The use of plasma discharge to improve various properties in low surface energy textile materials (e.g., polyethylene, polypropylene, and polyester) today is a deeprooted technology (De Geyter et al., 2008; Samanta et al., 2009; Leroux et al., 2009; Thurston et al., 2007; Aouinti et al., 2003; Kabajev and Prosycevas, 2004). The increase in surface wettability is certainly one of the simplest studied properties to identify surface modification of plasma-treated textile materials. However, plasma-surface interactions are not yet fully comprehended because they are influenced by complex factors such as the chemistry of plasma gases, the nature of the substrate, and the operating parameters. Moreover, the energy or power density is one of the most important parameters to calculate plasma treatment costs and benefits, and at the same time it is also the most omitted parameters in literature (Abd Jelil et al., 2013) (Table 6.2).

The most commonly used methodologies to hydrophobize a textile substrate can be divided into three categories: (1) plasma treatment, (2) plasma etching (or ablation), and (3) plasma polymerization (Roth, 2001; Bahners et al., 2008). Plasma treatment uses inert gases such as argon (Ar), helium $(\mathrm{He})$, nitrogen $\left(\mathrm{N}_{2}\right)$, and chemically active molecules such as oxygen $\left(\mathrm{O}_{2}\right)$ or ammonia $\left(\mathrm{NH}_{3}\right)$, as well as fluorinated gases such as carbon tetrafluoride $\left(\mathrm{CF}_{4}\right)$, hexafluoroethane $\left(\mathrm{C}_{2} \mathrm{~F}_{6}\right)$, perflutren $\left(\mathrm{C}_{3} \mathrm{~F}_{8}\right)$, perfluoroisobutylene $\left(\mathrm{C}_{4} \mathrm{~F}_{8}\right)$, decafluorocyclopentane $\left(\mathrm{C}_{5} \mathrm{~F}_{10}\right)$, trifluoromethane $\left(\mathrm{CHF}_{3}\right)$, sulfur hexafluoride $\left(\mathrm{SF}_{6}\right)$, and other (larger size) fluorine-containing molecules such as perfluoroalkyl acrylates (Zille et al., 2015; Morent et al., 2008; Yim et al., 2013; Gotoh et al., 2017; Vietro et al., 2015; Tendero et al., 2006; 
Table 6.3 List of most important input chemical monomers used in plasma hydrophobization treatment of textiles.

\begin{tabular}{|c|c|}
\hline $\begin{array}{l}\text { Atmospheric plasma } \\
\text { Refs: (Yim et al., 2013); (Gotoh et al., } \\
\text { 2017); (Tendero et al., 2006); (Zille et al., } \\
\text { 2015); (Sparavigna, 2008); (Morent et al., } \\
\text { 2008) }\end{array}$ & $\begin{array}{l}\text { Low pressure plasma } \\
\text { Refs: (Vietro et al., 2015); (Zille et al., } \\
\text { 2015); (Sparavigna, 2008); (Morent et al., } \\
\text { 2008); (Jafari et al., 2013); (Hochart et al., } \\
\text { 2003b); (Hegemann, 2006) }\end{array}$ \\
\hline $\mathrm{C}_{11} \mathrm{H}_{7} \mathrm{~F}_{13} \mathrm{O}_{2}$ & $\mathrm{CF}_{4}$ \\
\hline $\mathrm{C}_{13} \mathrm{H}_{7} \mathrm{~F}_{17} \mathrm{O}_{2} / \mathrm{C}_{15} \mathrm{H}_{7} \mathrm{~F}_{21} \mathrm{O}_{2}$ & $\mathrm{C}_{2} \mathrm{~F}_{4}$ \\
\hline Unidyne TG-571® & $\mathrm{C}_{3} \mathrm{~F}_{6}$ \\
\hline $\mathrm{CF}_{4}$ & $\mathrm{C}_{2} \mathrm{~F}_{6}$ \\
\hline $\mathrm{CF}_{3} \mathrm{CHF}_{2}$ & $\mathrm{C}_{3} \mathrm{~F}_{8}$ \\
\hline $\mathrm{CHF}_{3}$ & $\mathrm{C}_{4} \mathrm{~F}_{10}$ \\
\hline $\mathrm{C}_{3} \mathrm{~F}_{6}$ & $\mathrm{C}_{6} \mathrm{~F}_{14}$ \\
\hline $\mathrm{C}_{2} \mathrm{~F}_{6}$ & $\mathrm{C}_{4} \mathrm{~F}_{8}$ \\
\hline $\mathrm{C}_{8} \mathrm{~F}_{17} \mathrm{CH}_{2} \mathrm{CH}_{2} \mathrm{OCOCH}=\mathrm{CH}_{2}$ & $\mathrm{CF}_{3} \mathrm{CHF}_{2}$ \\
\hline $\mathrm{C}_{3} \mathrm{~F}_{8}$ & $\mathrm{SF}_{6}$ \\
\hline $\mathrm{C}_{13} \mathrm{H}_{7} \mathrm{~F}_{17} \mathrm{O}_{2}$ & $\mathrm{CF}_{3} \mathrm{SO}_{3} \mathrm{H}$ (co-monomer) \\
\hline $\mathrm{SF}_{6}$ & $\mathrm{C}_{2} \mathrm{ClF}_{3}$ (co-monomer) \\
\hline $\mathrm{H}_{2} \mathrm{C}=\mathrm{CHCO}_{2} \mathrm{CH}_{2} \mathrm{CH}_{2}\left(\mathrm{CF}_{2}\right)_{7} \mathrm{CF}_{3}$ & $\mathrm{C}_{6} \mathrm{~F}_{6}$ (co-monomer) \\
\hline $\mathrm{C}_{6} \mathrm{H}_{13} \mathrm{~F}_{3} \mathrm{O}_{3} \mathrm{Si}(\mathrm{FAS}-3)$ & $\mathrm{HC}_{6} \mathrm{~F}_{5}$ (co-monomer) \\
\hline C6F5Si(OC2H5)3 (FAS-5) & $\mathrm{CF}_{3}\left(\mathrm{CF}_{2}\right)_{7} \mathrm{CH}=\mathrm{CH}_{2}$ \\
\hline $\mathrm{C}_{13} \mathrm{H}_{13} \mathrm{~F}_{17} \mathrm{O}_{3} \mathrm{Si}$ (FAS-17) & $1,1,2,2$, tetrahydroperfluorodecyl acrylate \\
\hline
\end{tabular}

Sparavigna, 2008; Jafari et al., 2013). The plasma-activated gases introduce chemical functionalities or create and deposit free radicals onto the target surface that can be subsequently used to cross-link or surface-graft other molecules to attain specific surface properties (very often more hydrophilic surfaces).

Another method consists of the immersion of the fabric in a fluid of hydrophobic fluorinated prepolymer with added initiators followed by a plasma treatment leading to the grafting on the surface of the fabric. Plasma etching occurs when the substrate is bombarded with ions from the plasma to clean, sterilize, or enhance surface adhesion of the fabrics. For example, dry plasma etching can be accomplished by using $\mathrm{CF}_{4}$ in a plasma discharge to create active species capable of reacting chemically with the layer to be etched (Sigurdsson and Shishoo, 1997).

Plasma polymerization is a process where a monomer in vapor phase such as $\mathrm{CF}_{4}$, $\mathrm{C}_{2} \mathrm{~F}_{6}, \mathrm{C}_{3} \mathrm{~F}_{8}$, or larger fluorinated molecules such as fluorodecylacrylate is converted into reactive fragments, which polymerize at the surface (plasma-induced 
polymerization) or combine with polymers in the gas phase (plasma-state polymerization) to be deposited on the substrate (Sigurdsson and Shishoo, 1997; Li and Jinjin, 2007; Artus et al., 2012; Sun and Stylios, 2006). The deposition can occur while the plasma is excited or in a two-step process: (i) creation of radicals on the fiber surface by plasma in inert gas (e.g., argon) and (ii) reaction of these radicals with unsaturated monomers (Morent et al., 2008).

Numerous studies with different plasma discharges were conducted with the objective of increasing the hydrophobicity of various textile fibers such as polyesters, polyacrylonitrile, polypropylene, cotton, and silk. (Sigurdsson and Shishoo, 1997; Hegemann, 2005; Ji et al., 2008; Leroux et al., 2008; Hochart et al., 2003a; Höcker, 2002; Mattheus, 2005; Kim et al., 2006; Caschera et al., 2013; Vasiljević et al., 2012; Suanpoot et al., 2008). Nowadays, fluorocarbons are used to reduce the fiber friction due to their low frictional coefficients and hydrophobic properties. Despite the price of these fibers remains high, fluorocarbon fibers remain the most efficient solution to provide effective anti-stain to both water and oil at the same time (Bertaux et al., 2009).

The majority of plasma-based textile treatment processes for the production of hydrophobic and oleophobic surfaces (but also for some polymer coating, flame retardant, and medical antimicrobial fabrics) reported in the technical literature are based on nonthermal plasmas generated at low pressure (between 1 mTorr and 1 Torr) and in few cases at atmospheric pressure (Table 6.3). However, atmospheric plasma source designs based on corona discharges, glow discharges, dielectric barrier discharges (DBDs), plasma jet, capacitive or inductive coupled discharges, and radio frequency - or microwave-induced discharges have been intensively studied (Zille et al., 2015; Morent et al., 2008; Gotoh et al., 2017; Vietro et al., 2015; Tendero et al., 2006; Sparavigna, 2008; Jafari et al., 2013; Sigurdsson and Shishoo, 1997). Most of these technologies are still at an emerging stage although some of the manufacturers have developed commercial scale machinery and applications for specialized textiles that are currently being implemented at industrial scale.

\subsection{Other applications and barriers}

Compared with the traditional finishing processes, plasma technology has several advantages of improving the surface functionality, enhancing the interaction between fibers and reducing the usage of chemicals and energy, hence, cost saving. Plasma technology can be applied to fibers, yarns, fabrics, and garments; nonwovens; coated fabrics; and composites to enhance surface functionality. Plasma technology has the potential to be applied in a range of areas which includes antimicrobial treatment, UV resistant finish, self-cleaning, and flame retardant finish. Textiles with higher durability and fastness properties can be achieved with this technology without changing the textile bulk properties. The other areas of plasma application includes:

* Removing surface hairiness of textile materials.

* Scouring of cellulosic materials and synthetic materials such as polyester and nylon.

* Enhancing the electrical conductivity of yarns by plasma deposition. 
* Antishrink treatment of textiles.

* Improved adhesion between various textile substrates.

* Desizing of textile materials.

* Producing stain-resistant finishing in the fabrics.

* Silicone coating on textile substrates such as airbags.

* Reducing color variation of textile materials.

Plasma technology has some barriers that need to be addressed in order to make this technology successful. The major problem is the availability of commercial machinery for industrial applications, gaps in the applied research to commercialize the technology, slow development in industrial system, and less transparency of the results achieved. Although the cost of running the machinery is low in plasma technology, the initial invest will be high, which restricts the plasma application of textiles. Furthermore, the whole procedure and components must be carefully selected and monitored during the processing. Continuous processing of some plasma treatment can cause technical problems, which also needs to be addressed. Some low-pressure plasma systems are available in the European market. However, in many other countries, there are not many manufacturers of industrial machinery.

\subsection{Conclusions}

The application of plasma technology into fashion and textiles is gaining impetus due to its eco-friendliness. Plasma technology is a dry, nonpolluting and worker-friendly method to achieve surface functionality of fashion and textile materials without modifying the bulk properties of the materials. For textile applications, atmospheric nonthermal plasma application is best suited as most textile materials are heat-sensitive polymers. In the last decade or so, plasma technology has become a very active, high growth research field, due to its great potential for the surface modification of textile substrates in the textile industry.

The introduction of plasma treatment in textile industry has improved the sustainability factor, since plasma technology helps to reduce the use of water and chemicals, which are essential in traditional processes. It is very effective with many properties modification without affecting bulk properties of the textiles. Moreover, economywise, plasma treatment gains huge advantages over conventional wet methods with reduction in cost and energy. On the other hand, initial purchases of the system, especially the vacuum pumps are fairly expensive and it must go through meticulous inspections for optimal outcome. With all the benefits plasma technology convey, its potential in the future is very promising.

Several research studies have been done in the last couple of decades on the low-pressure plasma applications to achieve various functionalities. A range of fibrous materials including plastics, polymers, resins, metals, ceramics, bio- and inorganic materials have been intensively researched and produced promising results. The surface modification include hydrophilicity, hydrophobicity, adhesion, sterilization, chemical resistivity, and inertness. Plasma technology has a promising future to achieve technical results economically. 
Despite the high potential advantages and the environmentally friendly approach, the application possibilities of plasma technology in textile industry is still limited due to potential problems. The major problem is the availability of commercial machinery or industrial application systems, gaps in the applied research, and less transparency of the results achieved. In future, the textile and fashion manufacturers in developing countries will adopt this technology when these problems are overcome.

\section{References}

Abd Jelil, R., et al., 2013. Modeling plasma surface modification of textile fabrics using artificial neural networks. Eng. Appl. Artif. Intell. 26 (8), 1854-1864.

Akishev, Y.S., et al., 2003. One-atmosphere argon dielectric-barrier corona discharge as an effective source of cold plasma for the treatment of polymer films and fabrics. High Energy Chem. 37 (5), 286-291.

Aouinti, M., Bertrand, P., Poncin-Epaillard, F., 2003. Characterization of polypropylene surface treated in a $\mathrm{CO}_{2}$ plasma. Plasma Polym. 8 (4), 225-236.

Artus, G.R.J., et al., 2012. A superoleophobic textile repellent towards impacting drops of alkanes. Appl. Surf. Sci. 258 (8), 3835-3840.

Bahners, T., et al., 2008. Recent approaches to highly hydrophobic textile surfaces. J. Adhes. Sci. Technol. 22 (3-4), 285-309.

Barani, H., Maleki, H., 2011. Plasma and ultrasonic process in dyeing of wool fibers with madder in presence of lecithin. J. Dispersion Sci. Technol. 32 (8), 1191-1199.

Bertaux, E., et al., 2009. Effects of siloxane plasma coating on the frictional properties of polyester and polyamide fabrics. Surf. Coat. Technol. 204 (1-2), 165-171.

Bessada, R., et al., 2011. Functionalization of PET and PA6.6 woven fabrics. Appl. Surf. Sci. 257 (18), 7944-7951.

Bhat, N., et al., 2011. Surface modification of cotton fabrics using plasma technology. Text. Res. J. 81 (10), 1014-1026.

Binias, D., Wlochowicz, A., Binias, W., 2004. Selected properties of wool treated by lowtemperature plasma. Fibres Text. East. Eur. 12 (2), 58-62.

Borcia, G., Dumitrascu, N., Popa, G., 2005. Influence of dielectric barrier discharge treatments on the surface properties of polyamide-6 films. J. Optoelectron. Adv. Mater. 7 (5), 2535-2538.

Borcia, G., Anderson, C.A., Brown, N.M.D., 2006. Surface treatment of natural and synthetic textiles using a dielectric barrier discharge. Surf. Coat. Technol. 201 (6), 3074-3081.

Bradley, A., Fales, J.D., 1971. Prospects for industrial applications of electrical discharge. Chem. Technol. 232.

Bremen Cotton Report No. 05-06, 2019. Bremer Baumwollbörse, 28067 Bremen, Wachtstrasse 17-24, 28195 Bremen.

Cai, Z., Qiu, Y., 2008. Dyeing properties of wool fabrics treated with atmospheric pressure plasmas. J. Appl. Polym. Sci. 109 (2), 1257-1261.

Canal, C., et al., 2007. Role of the active species of plasmas involved in the modification of textile materials. Plasma Process. Polym. 4 (4), 445-454.

Carneiro, N., et al., 2001. Dyeability of corona-treated fabrics. Color. Technol. 117 (5), 298-302. 
Carneiro, N., Souto, A.P., Rios, M.J., 2005. Evaluation of cotton fabric properties after mercerisation, using CORONA discharge as a preparation step. In: 5th International Istanbul Textile Conference - Recent Advances in Innovation and Enterprise in Textiles and Clothing. Istanbul, Turquia.

Carneiro, N., et al., 2006. Preparation of cotton materials using corona discharge. J. Nat. Fibers 2 (4), 53-65.

Caschera, D., et al., 2013. Ultra hydrophobic/superhydrophilic modified cotton textiles through functionalized diamond-like carbon coatings for self-cleaning applications. Langmuir 29 (8), 2775-2783.

Cheng, C., Zhang, L.Y., Zhan, R.J., 2006. Surface modification of polymer fibre by the new atmospheric pressure cold plasma jet. Surf. Coat. Technol. 200 (24), 6659-6665.

Cheng, S.Y., et al., 2010. Influence of atmospheric pressure plasma treatment on various fibrous materials: performance properties and surface adhesion analysis. Vacuum 84 (12), 1466-1470.

Chvalinova, R., Wiener, J., 2008. Sorption properties of wool fibres after plasma treatment. Chem. Listy 102, S1473-S1477.

Costa, T.H.C., et al., 2006. Effects of gas composition during plasma modification of polyester fabrics. J. Mater. Process. Technol. 173 (1), 40-43.

De Geyter, N., Morent, R., Leys, C., 2008. Surface characterization of plasma-modified polyethylene by contact angle experiments and ATR-FTIR spectroscopy. Surf. Interface Anal. 40 (3-4), 608-611.

Demir, A., et al., 2011. Effect of the atmospheric plasma treatment parameters on jute fabric: the effect on mechanical properties of jute fabric/polyester composites. J. Appl. Polym. Sci. 121 (2), 634-638.

Deshmukh, R., Bhat, N., 2011. Pretreatments of Textiles Prior to Dyeing: Plasma Processing. Textile Dyeing.

ECHA Web Page, 2019. Available from: https://echa.europa.eu/reach-2018.

El-Nagar, K., et al., 2006. DC pseudo plasma discharge treatment of polyester textile surface for disperse dyeing. J. Text. Inst. 97 (2), 111-117.

El-Zawahry, M.M., Ibrahim, N.A., Eid, M.A., 2006. The impact of nitrogen plasma treatment upon the physical-chemical and dyeing properties of wool fabric. Polym. Plast. Technol. Eng. 45 (10), 1123-1132.

Fakin, D., Ojstrsek, A., Benkovic, S.C., 2009. The impact of corona modified fibres' chemical changes on wool dyeing. J. Mater. Process. Technol. 209 (1), 584-589.

Fang, K.J., Zhang, C.M., 2009. Surface physical-morphological and chemical changes leading to performance enhancement of atmospheric pressure plasma treated polyester fabrics for inkjet printing. Appl. Surf. Sci. 255 (17), 7561-7567.

Ferrero, F., et al., 2004. Improving the dyeability of synthetic fabrics with basic dyes using in situ plasma polymerisation of acrylic acid. Color. Technol. 120 (1), 30-34.

Freitas, D., et al., 2006. Tratamento em plasma de tecido $100 \%$ aramida para análise de molhabilidade. Rev. Bras. Apl. Vácuo 25 (4), 215-218.

Gawish, S.M., et al., 2011. The effect of low-temperature plasma for improving wool and chitosan-treated wool fabric properties. J. Text. Inst. 102 (2), 180-188.

Ghoranneviss, M., et al., 2011. Influence of plasma sputtering treatment on natural dyeing and antibacterial activity of wool fabrics. Prog. Org. Coat. 70 (4), 388-393.

Gleizes, A., Gonzalez, J.J., Freton, P., 2005. Thermal plasma modelling. J. Phys. D Appl. Phys. 38 (9), R153-R183.

Gorensek, M., et al., 2009. Functionalization of PET fabrics by corona and nano silver. Text. Res. J. 80 (3), 253-262. 
Gotoh, K., Yasukawa, A., 2010. Atmospheric pressure plasma modification of polyester fabric for improvement of textile-specific properties. Text. Res. J. 81 (4), 368-378.

Gotoh, K., Shohbuke, E., Ryu, G., 2017. Application of atmospheric pressure plasma polymerization for soil guard finishing of textiles. Text. Res. J. 88 (11), 1278-1289.

Hegemann, D., 2005. Stain repellent finishing on fabrics. Adv. Eng. Mater. 7 (5), 401-404.

Hegemann, D., 2006. Plasma polymerization and its applications in textiles. Indian J. Fibre Text. Res. 31, 99-115.

Hochart, F., De Jaeger, R., Levalois-Grutzmacher, J., 2003. Graft-polymerization of a hydrophobic monomer onto PAN textile by low-pressure plasma treatments. Surf. Coat. Technol. 165 (2), 201-210.

Hochart, F., De Jaeger, R., Levalois-Grützmacher, J., 2003. Graft-polymerization of a hydrophobic monomer onto PAN textile by low-pressure plasma treatments. Surf. Coat. Technol. 165 (2), 201-210.

Höcker, H., 2002. Plasma treatment of textile fibers. Pure Appl. Chem. 74 (3), 423-427.

Hossain, M.M., Herrmann, A.S., Hegemann, D., 2007. Plasma deposition of amine-embedded nanoporous ultrathin films on polyester enables substrate independent surface dyeing. Plasma Process. Polym. 4 (S1), S1068-S1074.

Hossain, M.M., et al., 2009. Ammonia/acetylene plasma deposition: an alternative approach to the dyeing of poly(ethylene terephthalate) fabrics at low temperatures. J. Appl. Polym. Sci. 111 (5), 2545-2552.

Huang, C.-Y., et al., 2013. Effects of argon plasma treatment on the adhesion property of ultra high molecular weight polyethylene (UHMWPE) textile. Surf. Coat. Technol. 231, 507-511.

Hwang, Y.J., et al., 2005. Effects of helium atmospheric pressure plasma treatment on low-stress mechanical properties of polypropylene nonwoven fabrics. Text. Res. J. 75 (11), 771-778.

Iriyama, Y., et al., 2002. Plasma treatment of silk fabrics for better dyeability. J. Photopolym. Sci. Technol. 15 (2), 299-306.

Jafari, R., Asadollahi, S., Farzaneh, M., 2013. Applications of plasma technology in development of superhydrophobic surfaces. Plasma Chem. Plasma Process. 33 (1), 177-200.

Ji, Y.Y., et al., 2008. Formation of hydrophobic and water-repellent surface on polyester fibers using Ar/hexamethyldisiloxane plasma at atmospheric pressure. Jpn. J. Appl. Phys. 47 (6), 4687-4691.

Jia, C., et al., 2011. Surface treatment of aramid fiber by air dielectric barrier discharge plasma at atmospheric pressure. Appl. Surf. Sci. 257 (9), 4165-4170.

Jin, J.C., Dai, J.J., 2003. Dyeing behaviour of nitrogen, low-temperature glow discharge treated wool. Indian J. Fibre Text. Res. 28 (4), 477-479.

Jocic, D., et al., 2005. Effect of low-temperature plasma and chitosan treatment on wool dyeing with Acid Red 27. J. Appl. Polym. Sci. 97 (6), 2204-2214.

Kabajev, M., Prosycevas, I., 2004. Plasma modification of structure and some properties of polyethylene therepthalate films and fibers. Mater. Sci. (Medžiagotyra) 10 (2), 173-176.

Kale, K.H., Desai, A.N., 2011. Atmospheric pressure plasma treatment of textiles using nonpolymerising gases. Indian J. Fiber Text. Res. 36 (September), 289-299.

Kamel, M.M., et al., 2011. Improvements in the dyeability of polyester fabrics by atmospheric pressure oxygen plasma treatment. J. Text. Inst. 102 (3), 220-231.

Kan, C.W., 2007. The use of plasma pre-treatment for enhancing the performance of textile inkjet printing. J. Adhes. Sci. Technol. 21 (10), 911-921.

Kan, C.W., Yuen, C.W.M., 2013. Effect of atmospheric pressure plasma treatment on wettability and dryability of synthetic textile fibres. Surf. Coat. Technol. 228, S607-S610. 
Kan, C.W., Yuen, C.W.M., Tsoi, W.Y., 2011. Using atmospheric pressure plasma for enhancing the deposition of printing paste on cotton fabric for digital ink-jet printing. Cellulose 18 (3), 827-839.

Karahan, H.A., Özdogan, E., 2008. Improvements of surface functionality of cotton fibers by atmospheric plasma treatment. Fibers Polym. 9 (1), 21-26.

Karahan, H.A., et al., 2008. Effects of atmospheric plasma treatment on the dyeability of cotton fabrics by acid dyes. Color. Technol. 124 (2), 106-110.

Karthik, T., Murugan, R., Vijayan, M., 2013. Optimization of plasma treatment variables to improve the hydrophilicity of polylinen ${ }^{\circledR}$ fabrics. J. Text. Inst. 104 (5), 481-493.

Ke, G.Z., et al., 2008. Effects of corona discharge treatment on the surface properties of wool fabrics. J. Mater. Process. Technol. 207 (1-3), 125-129.

Kerkeni, A., et al., 2012. Dyeing of woven polyester fabric with curcumin: effect of dye concentrations and surface pre-activation using air atmospheric plasma and ultraviolet excimer treatment. Color. Technol. 128 (3), 223-229.

Kim, J.H., Liu, G.M., Kim, S.H., 2006. Deposition of stable hydrophobic coatings with in-line $\mathrm{CH}_{4}$ atmospheric rf plasma. J. Mater. Chem. 16 (10), 977-981.

Klenko, Y., Pćhal, J., Aubrecht, L., 2006. Application of dielectric barrier discharge for treatment of polyester fabric. Czech. J. Phys. 56 (S2), B837-B842.

Koszewska, M., 2018. Circular economy - challenges for the textile and clothing industry. Autex Res. J. 18 (4), 337-347.

Kutlu, B., Aksit, A., Mutlu, M., 2010. Surface modification of textiles by glow discharge technique: part II: low frequency plasma treatment of wool fabrics with acrylic acid. J. Appl. Polym. Sci. 116 (3), 1545-1551.

Lehocký, M., Mráček, A., 2006. Improvement of dye adsorption on synthetic polyester fibers by low temperature plasma pre-treatment. Czech. J. Phys. 56 (S2), B1277-B1282.

Leroux, F., et al., 2008. Fluorocarbon nano-coating of polyester fabrics by atmospheric air plasma with aerosol. Appl. Surf. Sci. 254 (13), 3902-3908.

Leroux, F., et al., 2009. Atmospheric air plasma treatment of polyester textile materials. Textile structure influence on surface oxidation and silicon resin adhesion. Surf. Coat. Technol. 203 (20-21), 3178-3183.

Li, S., Jinjin, D., 2007. Improvement of hydrophobic properties of silk and cotton by hexafluoropropene plasma treatment. Appl. Surf. Sci. 253 (11), 5051-5055.

Li, H., et al., 2009. Air dielectric barrier discharges plasma surface treatment of threedimensional braided carbon fiber reinforced epoxy composites. Surf. Coat. Technol. 203 (10-11), 1317-1321.

Liao, J.-D., et al., 2005. Hydrophilic treatment of the dyed nylon-6 fabric using high-density and extensible antenna-coupling microwave plasma system. Plasma Chem. Plasma Process. 25 (3), 255-273.

Maamoun, D., Ghalab, S., 2013. Plasma utilization for treating wool/polyester blended fabric to improve its printability. Indian J. Fibre Text. Res. 38 (2), 180-185.

Malek, R.M.A., Holme, I., 2003. The effect of plasma treatment on some properties of cotton. Iran. Polym. J. 12 (4), 271-280.

Masaeli, E., Morshed, M., Tavanai, H., 2007. Study of the wettability properties of polypropylene nonwoven mats by low-pressure oxygen plasma treatment. Surf. Interface Anal. 39 (9), 770-774.

Mattheus, S.R., 2005. Plasma aided finishing of textile materials. In: Fiber and Polymer Science. North Carolina State University.

Mirjalili, M., Karimi, L., 2013. The impact of nitrogen low temperature plasma treatment upon the physical-chemical properties of polyester fabric. J. Text. Inst. 104 (1), 98-107. 
Mohammad, H., Dirk, H., 2011. Substrate independent dyeing of synthetic textiles treated with low-pressure plasmas. In: Hauser, P.P. (Ed.), Textile Dyeing. InTech.

Morent, R., et al., 2007. Surface modification of non-woven textiles using a dielectric barrier discharge operating in air, helium and argon at medium pressure. Text. Res. J. 77 (7), 471-488.

Morent, R., et al., 2008. Non-thermal plasma treatment of textiles. Surf. Coat. Technol. 202 (14), 3427-3449.

Motaghi, Z., Shahidi, S.H., Wiener, J., 2009. Application of low temperature plasma on dye ability of wool with madder. J. Theor. Appl. Phys. (Iran. Phys. J.) 3 (2), 17-23.

Muthu, S.S., 2018. Sustainable Innovations in Textile Chemical Processes. Textile Science and Clothing Technology. Springer Nature Singapore Pte Ltd.

Naebe, M., et al., 2009. Effects of plasma treatment of wool on the uptake of sulfonated dyes with different hydrophobic properties. Text. Res. J. 80 (4), 312-324.

Nasadil, P., Benesovsky, P., 2008. Plasma in textile treatment. Chem. Listy 102, S1486-S1489.

Negulescu, I.I., et al., 2000. Characterizing polyester fabrics treated in electrical discharges of radio-frequency plasma. Text. Res. J. 70 (1), 1-7.

Nourbakhsh, S., et al., 2008. Dyeability improvement of cationized corona discharge treated cotton fabric. Asian J. Chem. 20 (5), 3543-3548.

Oktem, T., et al., 1999. Modification of polyester fabrics by in situ plasma or post-plasma polymerisation of acrylic acid. J. Soc. Dye. Colour. 115 (9), 274-279.

Oktem, T., et al., 2000. Modification of polyester and polyamide fabrics by different in situ plasma polymerization methods. Turk. J. Chem. 24 (3), 275-285.

Oktem, T., et al., 2002. Improvement in surface-related properties of poly(ethylene terephthalate)/cotton fabrics by glow-discharge treatment. Indian J. Fibre Text. Res. 27 (2), $161-165$.

Oliveira, F.R., Souto, A.P., Carneiro, N., 2009. Polyamide Dyeing with anionic dyes after DBD Treatment - the Process's optimization. In: World Textile Conference. Izmir, Turkey.

Oliveira, F.R., et al., 2010. Surface modification on polyamide 6.6 with double barrier discharge (DBD) plasma to optimise dyeing process by direct dyes. Mater. Sci. Forum 636-637, 846-852.

Oliveira, F.R., Souto, P., Carneiro, N., 2010. Aplicacão da descarga plasmática de dupla barreira diéletrica (DBD) em fibras têxteis hidrofóbicas - estudo da forca colorística. Redige 1 (1), 127-140.

Oliveira, F.R., Zille, A., Souto, A.P., 2014. Dyeing mechanism and optimization of polyamide 6,6 functionalized with double barrier discharge (DBD) plasma in air. Appl. Surf. Sci. 293, $177-186$.

Ozdogan, E., et al., 2002. A new approach for dyeability of cotton fabrics by different plasma polymerisation methods. Color. Technol. 118 (3), 100-103.

Pappas, D., et al., 2006. Surface modification of polyamide fibers and films using atmospheric plasmas. Surf. Coat. Technol. 201 (7), 4384-4388.

Pappas, D.D., et al., 2008. Chemical and morphological modification of polymers under a helium, Äìoxygen dielectric barrier discharge. Surf. Coat. Technol. 203 (5-7), 830-834.

Patiño, A., et al., 2011. Surface and bulk cotton fibre modifications: plasma and cationization. Influence on dyeing with reactive dye. Cellulose 18 (4), 1073-1083.

Payamara, J., et al., 2010. Effect of electron irradiation on dye and printability of polypropylene (PP) fabrics: a novel method for decoration of PP fabrics. J. Text. Inst. 101 (11), 988-995.

Persin, Z., et al., 2012. Characterisation of surface properties of chemical and plasma treated regenerated cellulose fabric. Text. Res. J. 82 (20), 2078-2089. 
Pichal, J., et al., 2004. Application of atmospheric corona discharge for PES fabric modification. Czech. J. Phys. 54 (S3), C828-C834.

Poll, H.U., Schladitz, U., Schreiter, S., 2001. Penetration of plasma effects into textile structures. Surf. Coat. Technol. 142, 489-493.

Radetic, M., et al., 2000. The effect of low-temperature plasma pretreatment on wool printing. Text. Chem. Am. Dyest. Report. 32 (4), 55-60.

Radetic, M., et al., 2007. Environmental impact of plasma application to textiles. In: First International Workshop on Nonequilibrium Processes in Plasma Physics and Studies of Environment, vol. 71, pp. U214-U224.

Raffaele-Addamo, A., et al., 2006. Cold plasma-induced modification of the dyeing properties of poly(ethylene terephthalate) fibers. Appl. Surf. Sci. 252 (6), 2265-2275.

Rahel', J., et al., 2003. Hydrophilization of polypropylene nonwoven fabric using surface barrier discharge. Surf. Coat. Technol. 169, 604-608.

Rashed, U.M., et al., 2009. Surface characteristics and printing properties of PET fabric treated by atmospheric dielectric barrier discharge plasma. Eur. Phys. J. Appl. Phys. 45 (1).

Ratnapandian, S., et al., 2011. Effect of atmospheric plasma treatment on pad-dyeing of natural dyes on wool. In: Textile Bioengineering and Informatics Symposium Proceedings, vols 1-3, pp. 780-786.

Ren, C.S., Wang, D.Z., Wang, Y.N., 2008. Improvement of the graft and dyeability of linen by DBD treatment in ambient air. J. Mater. Process. Technol. 206 (1-3), 216-220.

Ren, Y., Deng, J., Li, Z.H., 2011. Influence of the combined treatment of causticization and low temperature plasma on the properties of polyester fiber. In: Silk: Inheritance and Innovation - Modern Silk Road, vol. 175-176, pp. 312-317.

Riccardi, C., et al., 2003. Surface modification of poly(ethylene terephthalate) fibers induced by radio frequency air plasma treatment. Appl. Surf. Sci. 211 (1-4), 386-397.

Riccardi, C., Barni, R., Esena, P., 2005. Plasma treatment of silk. Solid State Phenom. 107, $125-128$.

Roth, J.R., 2001. Industrial Plasma Engineering: Volume 2 - Applications to Nonthermal Plasma Processing, vol. 2. CRC press.

Ryu, J., Wakida, T., Takagishi, T., 1991. Effect of corona discharge on the surface of wool and its application to printing. Text. Res. J. 61 (10), 595-601.

Salem, T., et al., 2011. Modification of plasma pre-treated PET fabrics with poly-DADMAC and its surface activity towards acid dyes. Prog. Org. Coat. 72 (1-2), 168-174.

Samanta, K.K., Jassal, M., Agrawal, A.K., 2009. Improvement in water and oil absorbency of textile substrate by atmospheric pressure cold plasma treatment. Surf. Coat. Technol. 203 (10-11), 1336-1342.

Sarmadi, A.M., Ying, T.H., Denes, F., 1993. Surface modification of polypropylene fabrics by acrylonitrile cold plasma. Text. Res. J. 63 (12), 697-705.

Sarmadi, M., Denes, A.R., Denes, F., 1996. Improved dyeing properties of SiCi(4) (ST)-plasma treated polyester fabrics. Text. Chem. Colorist 28 (6), 17-22.

Sarra-Bournet, C., et al., 2006. A study of atmospheric pressure plasma discharges for surface functionalization of PTFE used in biomedical applications. J. Phys. D Appl. Phys. 39 (16), 3461-3469.

Schneider, K., Hafner, C., Jäger, I., 2004. Mutagenicity of textile dye products. J. Appl. Toxicol. 24 (2), 83-91.

Shah, J., Shah, S., 2013. Innovative plasma technology in textile processing: a step towards green environment. Res. J. Eng. Sci. 2278, 9472.

Shahidi, S., et al., 2007. Effect of using cold plasma on dyeing properties of polypropylene fabrics. Fibers Polym. 8 (1), 123-129. 
Sigurdsson, S., Shishoo, R., 1997. Surface properties of polymers treated with tetrafluoromethane plasma. J. Appl. Polym. Sci. 66 (8), 1591-1601.

Simor, M., et al., 2010. The influence of surface DBD plasma treatment on the adhesion of coatings to high-tech textiles. J. Adhes. Sci. Technol. 24 (1), 77-97.

Souto, A.P., Ribeiro, F., Carneiro, N., 2011. Polyamide 6.6 modified by DBD plasma treatment for anionic dyeing processes. In: Hauser, P.J. (Ed.), Texile Dyeing. Intech, pp. 241-260.

Souto, A.P., et al., 2012. Poliamid Boyama İşleminde DBD Plazma Modifikasyonunun Etkisi. Tekstil ve Mühendis, pp. 20-25.

Sparavigna, A., 2008. Plasma Treatment Advantages for Textiles arXiv preprint arXiv: 0801.3727.

Suanpoot, P., et al., 2008. Surface analysis of hydrophobicity of Thai silk treated by SF6 plasma. Surf. Coat. Technol. 202 (22-23), 5543-5549.

Sun, S., Qiu, Y., 2012. Influence of moisture on wettability and sizing properties of raw cotton yarns treated with $\mathrm{He} / \mathrm{O}_{2}$ atmospheric pressure plasma jet. Surf. Coat. Technol. 206 (8-9), 2281-2286.

Sun, D., Stylios, G.K., 2004. Effect of low temperature plasma treatment on the scouring and dyeing of natural fabrics. Text. Res. J. 74 (9), 751-756.

Sun, D., Stylios, G.K., 2006. Fabric surface properties affected by low temperature plasma treatment. J. Mater. Process. Technol. 173 (2), 172-177.

Temmerman, E., Leys, C., 2005. Surface modification of cotton yarn with a DC glow discharge in ambient air. Surf. Coat. Technol. 200 (1-4), 686-689.

Tendero, C., et al., 2006. Atmospheric pressure plasmas: a review. Spectrochim. Acta B At. Spectrosc. 61 (1), 2-30.

Thurston, R.M., Clay, J.D., Schulte, M.D., 2007. Effect of atmospheric plasma treatment on polymer surface energy and adhesion. J. Plastic Film Sheeting 23 (1), 63-78.

Tian, L., et al., 2011. Helium/oxygen atmospheric pressure plasma jet treatment for hydrophilicity improvement of grey cotton knitted fabric. Appl. Surf. Sci. 257 (16), 7113-7118.

Upadhyay, D.J., et al., 2004. A comparative study of the surface activation of polyamides using an air dielectric barrier discharge. Colloid. Surf. Physicochem. Eng. Asp. 248 (1-3), 47-56.

Vander Wielen, L.C., et al., 2006. Surface modification of cellulosic fibers using dielectricbarrier discharge. Carbohydr. Polym. 65 (2), 179-184.

Vasiljević, J., et al., 2012. The surface modification of cellulose fibres to create superhydrophobic, oleophobic and self-cleaning properties. Cellulose 20 (1), 277-289.

Vatuňa, T., et al., 2004. PES fabric plasma modification. Czech. J. Phys. 54 (S3), C475-C482.

Verschuren, J., 2005. Influence of fiber surface purity on wicking properties of needle-punched nonwoven after oxygen plasma treatment. Text. Res. J. 75 (5), 437-441.

Vietro, N.D., et al., 2015. Plasma treatment for preparing durable water repellent and anti-stain synthetic fabrics for automotive applications. J. Surf. Eng. Mater. Adv. Technol. 5 (3), $103-109$.

Wang, C.X., Qiu, Y.P., 2007. Two sided modification of wool fabrics by atmospheric pressure plasma jet: influence of processing parameters on plasma penetration. Surf. Coat. Technol. 201 (14), 6273-6277.

Wang, C., Qiu, Y., 2012. Study on wettability improvement and its uniformity of wool fabric treated by atmospheric pressure plasma jet. J. Appl. Polym. Sci. 123 (2), 1000-1006.

Wang, C.Y., Wang, C.X., 2010. Surface pretreatment of polyester fabric for ink jet printing with radio frequency O-2 plasma. Fibers Polym. 11 (2), 223-228.

Wang, C.X., et al., 2008. Influence of atmospheric pressure plasma treatment time on penetration depth of surface modification into fabric. Appl. Surf. Sci. 254 (8), 2499-2505. 
Wei, Q.F., et al., 2005. Functional nanostructures generated by plasma-enhanced modification of polypropylene fibre surfaces. J. Mater. Sci. 40 (20), 5387-5392.

Xi, M., et al., 2008. Surface modification of aramid fiber by air DBD plasma at atmospheric pressure with continuous on-line processing. Surf. Coat. Technol. 202 (24), 6029-6033.

Xiaoliang, T., et al., 2007. Dyeing behavior of atmospheric dielectric barrie discharge $\mathrm{Ar}-\mathrm{O}_{2}$ plasma treated poly(ethylene terephthalate) fabric. In: ICOPS 2007. IEEE 34th International Conference on Plasma Science. Albuquerque, USA.

Xu, W., Liu, X., 2003. Surface modification of polyester fabric by corona discharge irradiation. Eur. Polym. J. 39 (1), 199-202.

Yaman, N., et al., 2009. Plasma treatment of polypropylene fabric for improved dyeability with soluble textile dyestuff. Appl. Surf. Sci. 255 (15), 6764-6770.

Yaman, N., Özdoğan, E., Seventekin, N., 2011. Atmospheric plasma treatment of polypropylene fabric for improved dyeability with insoluble textile dyestuff. Fibers Polym. 12 (1), 35-41.

Yaman, N., Özdoğan, E., Seventekin, N., 2013. Effect of surrounded air atmospheric plasma treatment on polypropylene dyeability using cationic dyestuffs. Fibers Polym. 14 (9), $1472-1477$.

Yim, J.H., et al., 2013. Atmospheric pressure plasma enhanced chemical vapor deposition of hydrophobic coatings using fluorine-based liquid precursors. Surf. Coat. Technol. 234, 21-32.

Yip, J., et al., 2002. Study of physico-chemical surface treatments on dyeing properties of polyamides. Part 1: effect of tetrafluoromethane low temperature plasma. Color. Technol. 118 (1), 26-30.

Yuen, C.W.M., Kan, C.W., 2007. Influence of low-temperature plasma on the ink-jet-printed cotton fabric. J. Appl. Polym. Sci. 104 (5), 3214-3219.

Zhang, C.M., Fang, K.J., 2009. Surface modification of polyester fabrics for inkjet printing with atmospheric-pressure air/Ar plasma. Surf. Coat. Technol. 203 (14), 2058-2063.

Zhang, C.M., Fang, K.J., 2011. Influence of penetration depth of atmospheric pressure plasma processing into multiple layers of polyester fabrics on inkjet printing. Surf. Eng. 27 (2), 139-144.

Zille, A., Oliveira, F.R., Souto, A.P., 2015. Plasma treatment in textile industry. Plasma Process. Polym. 12 (2), 98-131. 